\title{
Probiotics as an additional therapeutic approach in the management of Type 2 Diabetes Mellitus: a systematic review and meta-analysis
}

\author{
L. Nejad ${ }^{1}$, S. Al-Naimi ${ }^{1}$, A. Rajic ${ }^{1}$ and F. Ibrahim ${ }^{1}$ \\ ${ }^{1}$ Life Sciences Directorate, University of Suffolk, Ipswich, Suffolk, UK
}

The incidence of type 2 diabetes mellitus (T2DM) is increasing worldwide. The treatment for T2DM includes a variety of anti-diabetic drugs, which together with changes in lifestyle factors, can help reduce the severity of the condition. Recent research has identified the role of an altered gut microbiota in the development of T2DM, and hence the manipulation of the gut microbiota through therapeutic dietary strategies could be factorised in the treatment of $T 2 \mathrm{DM}^{(1-4)}$. The aim of this systematic review and meta-analysis was to evaluate the efficacy of probiotics in the management of T2DM.

The online databases PubMed, the Cochrane Library (CENTRAL), ProQuest and Web of Science were chosen for the search. The search terms were formulated using the PICO(S) framework approach for this review as follows: [P] Population - patients with T2DM, [I] Intervention - probiotics (single or multispecies) in any form (supplement, capsule, sachet, food product: milk, yogurt), [C] Comparison - placebo (similar intervention without probiotic administration), [O] Outcomes - glycaemic parameters (FBG, HbA1c), insulin resistance and inflammatory markers (hs-CRP), [S] Study design - RCTs. The meta-analysis included 14 randomised controlled trials with a total of 841 patients with T2DM, during an 8-12-week period of probiotic consumption in various forms and doses. All T2DM patients were on a stable form of anti-diabetic medication. RCTs (published in English) that investigated the effect of probiotics on glycaemic parameters (FBG, HbA1c), HOMA-IR and inflammatory markers (TNF-a, IL-6 and hs-CRP), with T2DM participants of both sexes, aged $\geq 18$ years and had a BMI $>18.5-<35.0 \mathrm{~kg} / \mathrm{m}^{2}$, were included. The study duration of the included studies had to be more than 4 weeks, and participants were to carry on with their current lifestyle and dietary habits to ensure change in dietary habits did not influence the results.

Probiotics for the management of T2DM were shown to significantly improve the levels of fasting blood glucose by $-12.15 \mathrm{mg} / \mathrm{dL}$ $(\mathrm{p}=<0.0001)$, the glycated haemoglobin A1c levels by 0.31 percentage points $(\mathrm{p}=<0.01)$, and insulin resistance by 1.63 units $(\mathrm{p}=<0.001)$ compared to control group. Additionally, the level of high-sensitivity C-reactive protein decreased significantly with a mean difference of $0.26 \mathrm{mg} / \mathrm{L}(\mathrm{p}=0.03)$.

Probiotics appeared to have beneficial effects in the management of postprandial blood glucose levels and in reducing inflammatory markers. Further clinical studies with longer duration periods should be conducted to determine the long-term effects of probiotics as well as elucidating the mechanism of action.

\section{References}

1. Asemi Z. et al. (2013) Ann Nutr Metab 63, 1-9.

2. Feizollahzadeh S. et al. (2017) Probiotics Antimicrob Protein 9, 41-47.

3. Khalili L. et al. (2019) Iran Biomed J 23, 68-77.

4. Razmpoosh E. et al. (2019) Diabetes Metab Syndr 13, 175-182. 